sequently, it does not seem possible to interpret the decay curve of the nutation and to discriminate the effect of damping from the beat phenomenon.

\section{B. Gutenberg}

Seismological Laboratory,

California Institute of Technology,

Pasadena.

Dec. 27.

"Jeffreys, H., "The Earth", 3rd edit., 210, 244 (1952).

Bondi, H., and Gold, T., Mon. Not. Roy. Astro. Soc., 115, 41 (1955)

${ }^{3}$ Schweydar, W., Sitzungsber. Preuss. Akad. Wiss., 20, 357 (1919).

"Gutenberg, B., "Internal Constitution of the Earth", 2nd edit., 389 (1951).

\section{A New Theorem in Electrostatics and its Application to Calculable Standards of Capacitance}

Although capacitance has the dimensions of length only, usually two or more separate lengths are needed to compute the capacitance of the various forms that have been used as standards. In seeking a form of calculable capacitor involving a minimum number of determinations of length, we have investigated cylindrical systems and have discovered a particular class of capacitor in which the capacitance depends on one length only to the first order. For example, if a square cylinder is constructed from four conducting planes which are insulated from each other at the corners, then the direct capacitances per unit length of cylinder, between each pair of opposing inside faces, are equal and independent of the size of the square. This direct capacitance was calculated as $\frac{\ln 2}{4 \pi^{2}} \mathrm{~cm} . / \mathrm{cm}$., and it was also calculated that if the cross-section was rectangular with a ratio of sides of $1+\delta, \delta \ll 1$, then the mean of the two cross-capacitances was $\frac{\ln 2}{4 \pi^{2}}\left\{1+3 \cdot 45 \delta^{2}+\ldots\right\}$ cm. $/ \mathrm{cm}$. These calculations confirmed that, providing the asymmetry was not too great, a standard capacitor could be constructed which required only one length measurement for the computation. of its capacitance. The length of the capacitor may be defined by insulating a length of one face from the remainder of that face, which remainder functions as as guard.

The capacitance per unit length and asvmmetry effects were calculated for a number of other symmetrical cross-sections. These calculations all gave the same value, namely, $\frac{\ln 2}{4 \pi^{2}}$ e.s.u., as the capacitance

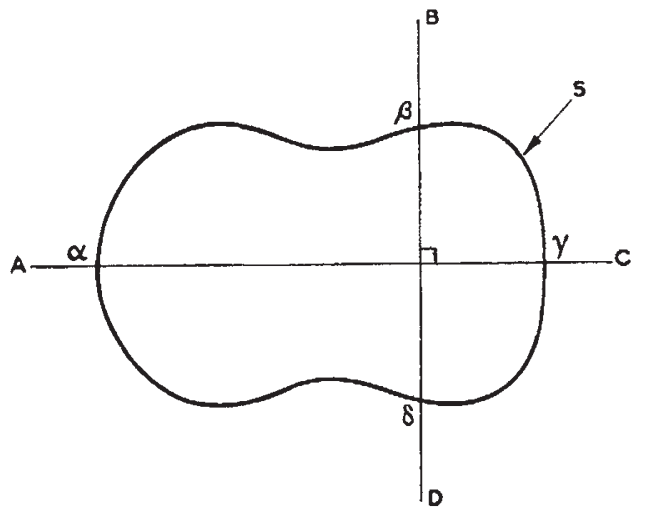

Fig. 1. Cross-section of cylindrical shell

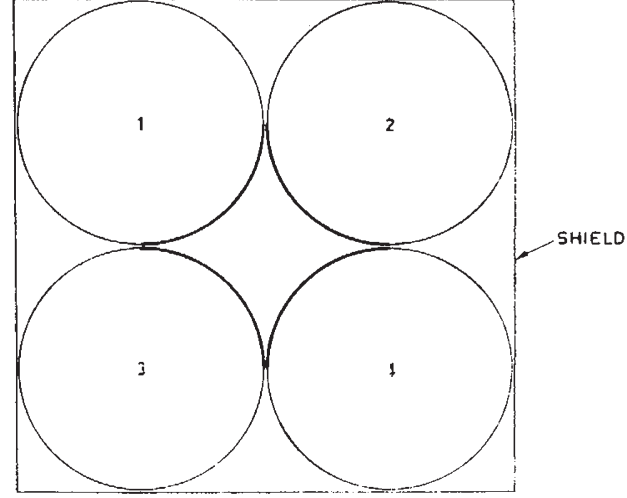

Fig. 2. (ross-scetion of experimental capacitor

per unit length, and this result led one of us (D. (X. L.) to propose and prove the following theorem:

Let the closed curve $S$ (see Fig. 1) be the crosssection of a conducting cylindrical shell, which crosssection has one axis of symmetry $A C$ but is otherwise arbitrary. Further, let this shell be divided into four parts by two planes at right angles, the line of intersection of the planes being parallel to the generators of the cylinder, and one of the planes containing the symmetry axis $A C$. Then the direct capacitance, per unit length of the cylinder, between opposing parts of the shell (for example, $\alpha \beta$ to $\gamma \delta$ ), due to the field inside (or outside) the shell, is a constant :

$$
C_{0}=\frac{\ln 2}{4 \pi^{2}} \text { e.s.u. }=0 \cdot 0175576 \text { e.s.u. }
$$

As a consequence of this theorom, it is clear that the choice of the cross-section, for a cylindrical standard capacitor, is limited only by the requirements that the cross-section have one axis of symmetry and that it be a closed curve.

A cross-section which satisfies these requirements and at the same time is easy to realize in practice is that of the space between four circular cylinders the axes of which are arranged to lie at tho corners of a square (see Fig. 2). Accordingly, a capacitor of nominal capacitance $1 \mathrm{pF}$ (equivalent length, 20-151 in.) was constructed, using four piecess of stock silver-steel rod (nominal diameter, 15/16 in.) for the cylinders and suitably shielded externally so that only the inside field contributes to the capacitance. Two of the rods ( 2 and 4 ) were provided with end-guards to define their lengths and the rods were insulated from each other by mica spacers $(0.004 \mathrm{in}$. thick $)$ placed in the end guard region.

The cross-capacitances (1-4 and 2-3) were measured, and although they differed from the mean capacitance by as much as $0 \cdot 3$ per cent, this mean agreed to within one part in ten thousand with the capacitance calculated from the measured lengths of the guardod rods, due allowance being made for the dielectric constant of tho air. It is proposed to construct a precision capacitor based on this design.

Full details of these investigations will be published elsewhere.

A. M. Thompson

Division of Electrotechnology, D. G. IJampard

Commonwealth Scientific and

Industrial Research Organization, University Grounds, Sydney. Nov. 2. 